[3] M. Kac, On some connections between probability theory and differential and integral equations, Proc. Second Berkeley Symposium (1951), pp. 189-215.

[4] H. P. McKean, Jr., Stochastic integrals, Academic Pross, 1969.

[5] F. G. Tricomi, Differential equations, Blackie \& Sons, 1961.

Added in proof: For results in $\boldsymbol{R}^{d}, d \geqslant 1$ see K. L. Chung and K. M. Rao, Sur la théorie du potentiel avec la fonetionnelle de F'oynman-Krac, Comp. Rend. Acąd. Sci., Paris 290 (31 mars 1980).

\section{DEPARTMENT OF MATHEMATIOS}

STANFORD UNIVERSTTY

\section{Multilinear singular integrals}

by

JONATHAN COHEN (Athens, Ga.)

Abstract. This paper uses Fourier Transform and Mellin Transform analysis to obtain $L^{p}$ estimates for certain multilinear singular integrals. The results obtained here extend estimates by Calderón, Coifman and Meyer on commutators of singular integrals to a wider class of multilinear singular integrals.

\$ 1. Introduction. In this paper sharp estimates are obtained for operators of the type:

$$
\text { p.v. } \int \prod_{j=1}^{n}\left\{\frac{r_{m_{j}}\left(A_{j} ; x, y\right)}{(x-y)^{m_{j}}}\right\} \frac{f(y)}{x-y} d y .
$$

where

$$
r_{m_{j}}\left(A_{j} ; x, y\right)=A_{j}(x)-\sum_{k=1}^{m_{j}-1} \frac{A_{j}^{(k)}(y)(x-y)^{k}}{k !} .
$$

These operators are related to those introduced by Calderón in [2] and [3] and studied by Coifman and Meyer in [5], [6] and [7]. We will sometimes denote these operators by $D^{N} H\left\{\prod_{j=1}^{n} r_{m_{j}}\left(A_{j} ; x, \cdot\right) f(\cdot)\right\}$ where $D^{N} H$ is the Hilbert transform followed by the $n$th derivative. (The reason for this notation will become apparent in §3.)

The operators studied in this paper arise naturally from the study of higher commutators of differential and pseudo-differential operators. The simplest case is the commutator $\left[A, D^{N} H\right]$ where $A$ is pointwise multiplication by the function $A(x)$. It has been shown by Calderón [3] that this commutator can be written as the sum of pseudo-differential operators of degree less than or equal to $N-1$ plus an operator of the type studied in this paper.

One can show that higher commutators of the form $\left[A_{1}, \ldots,\left[A_{n}, D^{N} H\right]\right.$ ...] can be written as the sum of pseudo-differential operators of degree less than or equal to $N-n$ plus the sum of operators of the type considered in this paper. One example is the second commutator $\left[A,\left[B, D^{N} H\right]\right]$ 
which can be written

$$
\begin{aligned}
{\left[A,\left[B, D^{N} H\right]\right] f=} & D^{N} H\left[r_{m}(A ; x, \cdot) r_{n}(B ; x, \cdot) f(\cdot)\right] \\
& +\sum_{k=1}^{n-1}(-1)^{k}\left(\begin{array}{l}
N \\
k
\end{array}\right) D^{N-k} H\left[r_{N-k_{0}}(A ; x, \cdot) B^{(k)}(\cdot) f(\cdot)\right] \\
& +\sum_{j=1}^{m-1}(-1)^{j}\left(\begin{array}{l}
N \\
j
\end{array}\right) D^{N-j} H\left[r_{N-j}(B ; x, \cdot) A^{(j)}(\cdot) f(\cdot)\right] \\
& +\sum_{t=2}^{N-1} \sum_{j+k=t}(-1)^{j+k}\left(\begin{array}{l}
N \\
j, l_{k}
\end{array}\right) D^{N-j-k} H\left[A^{(j)} B^{(k)} f\right]
\end{aligned}
$$

where $m+n=N, j \geqslant 1, k \geqslant 1$ and $\left(\begin{array}{l}N \\ j, k\end{array}\right)=\frac{N !}{j ! k !(N-j-k) !}$.

The results and methods used in this paper closely follow those developed by Coifman and Meyer in [6] and [7]. It appears as a natural follow up to [7] and the notation and organization have been chosen to be as consistent as possible with it.

Let $a_{j} \in \mathscr{S}, j=1, \ldots, n, f \in \mathscr{S}(\mathscr{S}$ is the space of functions which are $C^{\infty}$ and rapidly decreasing). Let $\sigma: R^{n+1} \rightarrow C$ be bounded and measurable. Assume $\alpha=\left(a_{1}, \ldots, \alpha_{n}\right) \in R^{n}, s \in R$ and $(\alpha, s)=\left(\alpha_{1}, \ldots, a_{n}, s\right)$ $\in R^{n+1}$. We adopt the notation $\sigma(\alpha, s)=\sigma\left(\alpha_{1}, \ldots, \alpha_{n}, s\right), \hat{a}(\alpha)=\prod_{j=1}^{n} \hat{a}_{j}\left(\alpha_{j}\right)$ and $d \alpha=\prod_{j=1}^{n} d \alpha_{j}$.

Let ${\stackrel{P}{P_{n}=1}}_{n}=\left\{\left(p_{1}, \ldots, p_{n}, p_{0}\right): 1<p_{j}<\infty ; \sum_{j=1}^{n}\left(1 / p_{j}\right)=1 / q<1\right\}$. For $\left(p, p_{0}\right)=\left(p_{1}, \ldots, p_{n}, p_{0}\right)$, we define

and

$$
L^{\left(p, p_{0}\right)}=\left\{(a, f): a_{j} \in L^{p_{j}}, j=1,2, \ldots, n, f \in L^{p_{0}}\right\},
$$

$$
\|(a, f)\|_{\left(p, p_{0}\right)}=\left\{\prod_{j=1}^{n}\left\|a_{j}\right\|_{p_{j}}\right\}\|f\|_{p_{0}} .
$$

Using the above notation and assumptions on the functions $a_{j}, f$ and $\sigma$ we make the following definitions.

(1.3) Definitron.

(1) $T_{\sigma}(\alpha, f)(x)=\int_{R^{n+1}} e^{i s x} \sigma(\alpha, s) \hat{a}(\alpha) \hat{f}\left(s-\sum_{j=1}^{n} a_{j}\right) d \alpha d s$.

(2) For $\left(p, p_{0}\right) \in P_{n}, \sum_{j=0}^{n}\left(1 / p_{j}\right)=\frac{1}{q}<1$,

$$
M_{n}\left(p, p_{0}\right)=\left\{\sigma:\left\|T_{\sigma}(a, f)\right\|_{q} \leqslant e\|(a, f)\|_{\left(p, p_{0}\right)}\right\}
$$

$$
M_{n}=\bigcap_{\left(p, p_{0}\right) \in P_{n}} M_{n}\left(p, p_{0}\right)
$$

The function $\sigma$ will be called the symbol of the operator $T_{\sigma}$.

For $g(x)$ a function $\left(x \in R^{1}\right)$ with $m \geqslant 1$ derivatives, we define the Taylor scries remainder operator

$$
R_{-\alpha}^{m} g(x)=g(s-\alpha)-\sum_{l=0=0}^{m-1} \frac{g^{(k)}(s)(-\alpha)^{k c}}{k !} .
$$

We let $R_{-\alpha}^{0} g(s)=g(s-\alpha)$

If $m=\left(m_{1}, \ldots, m_{n}\right) \in Z^{n}, 0 \leqslant m_{j}, \alpha \in R^{n}$ and $g$ has $|m|=\sum_{j=1}^{n} m_{j}$ derivatives, we denote the $n$-fold composition of Taylor series of $g$ by Let

$$
R_{(-a)}^{(m)} g(s)=R_{-\alpha_{1}}^{m_{1}} \ldots R_{-\alpha_{n}}^{m_{n}} g(s) .
$$

$$
\omega_{m}(\alpha, s)=\frac{R_{(-a)}^{(m)} s^{|m|} \operatorname{sgn} s}{\prod_{j=1}^{n} \alpha_{j}^{m_{j}}}
$$

The operator $T_{\omega_{m}}$ will be called a oommutator of order $|m|$. We note that if $m_{j}=1$ for $j=1,2, \ldots, n$, then the operator $T_{\omega_{m}}$ is the $n$th commutator treated in the papers of Doifman and Meyer [6] and [7]. (The order of the operator $T_{\omega}$ does not have the same meaning as the order of a psoudo-difforential operator.)

The main results of this paper are the following theorems.

THWORTM I. If $a_{1}, \ldots, a_{n} \in \mathscr{P}$, then

$$
\left\|T_{\omega_{m}}(a, f)\right\|_{\alpha} \leqslant c\|f\|_{p_{0}} \prod_{j=1}^{n}\left\|a_{j}\right\|_{p_{j}}
$$

where $1>1 / q=\sum_{j=0}^{n}\left(1 / p_{j}\right), 1<p_{j}<\infty, j=0,1, \ldots, n$.

THeorim. IT. For $a_{j} \in \mathscr{D}\left(0^{\infty}\right.$ functions with compact support), $a_{j}=$ $A_{j}^{\left(m_{j}\right)}=\left(\frac{d}{d x_{i}}\right)^{m_{j}} A_{j}$, and

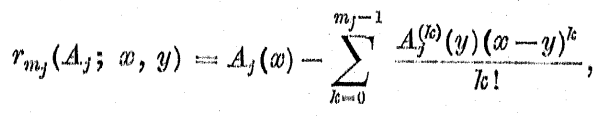

(1.4) p.v. $\int \prod_{j=a 1}^{n}\left\{\frac{r_{m_{j}}\left(A_{j j} ; x, y\right)}{(x-y)^{m_{j}}}\right\} \frac{f(y)}{(x-y)} d y$

$$
=c_{m} \int e^{i s x} \omega_{m}(\alpha, s) \hat{a}(\alpha) \hat{f}\left(s-\sum_{j=1}^{n} \alpha_{j}\right) d \alpha d s
$$

where $o_{m}=\frac{(-i \pi)(-1)^{|m|}}{|m| !(2 \pi)^{n+1}}$. 
THeorein III. We define $T_{\varepsilon}^{m}(a, f)=T_{\varepsilon}^{m_{1}, \ldots, m_{n}}\left(a_{1}, \ldots, a_{n}\right)$ by

$$
T_{\varepsilon}^{m}(a, f)(x)=\int_{|x-y|>\varepsilon} \prod_{j=1}^{n}\left\{\frac{r_{m_{j}}\left(A_{j} ; x, y\right)}{(x-y)^{m_{j}}}\right\} \frac{f(y)}{x-y} \cdot d y
$$

and let

$$
T_{*}^{m}(a, f)(x)=\sup _{e>0}\left|T_{\varepsilon}^{m}(a, f)(x)\right| .
$$

Then, for $0<q<\infty$

$$
\int\left|T_{*}^{m}(a, f)(x)\right|^{\alpha} d x \leqslant c \int\left\{\left[\prod_{j=1}^{n} a_{j}^{*}(x)\right] f^{* k}(x)\right\}^{\alpha} d x
$$

where $a_{j}^{*}$ is the Hardy-Littlewood maximal function of $a$.

§2. An $L^{p}$ estimate for smooth functions. We defined the order of the symbol $\omega_{m}$ to be $|m|=\sum_{j=1}^{n} m_{j}$ where $m=\left(m_{1}, \ldots, m_{n}\right) \in Z^{n}$ and $0 \leqslant m_{j}$. The proof of Theorem 1 is by induction on the order of $\omega_{m}$.

To clarify the inductive hypothesis we make the following observations.

(1) Let $\sigma\left(\alpha_{1}, \ldots, \alpha_{n}, s\right) \in M_{n}$. Then if $\tau\left(\alpha_{1}, \ldots, \alpha_{n}, \beta, s\right)=\sigma\left(\alpha_{1}, \ldots\right.$ $\left.\ldots, \alpha_{n}, s\right), \tau \in M_{n+1}$.

(2) If $\sigma \in M_{n}$, then $R_{-\beta}^{0} \sigma\left(\alpha_{1}, \ldots, \alpha_{n}, s\right)=\sigma\left(\alpha_{1}, \ldots, \alpha_{n}, s-\beta\right) \in M_{n+1}$.

(3) The zero order symbols are those $\omega^{\prime}$ s of the form $R_{-a_{1}}^{0} \ldots R_{-a_{n}}^{0} \operatorname{sgn} s$ $=\operatorname{sgn}\left(s-\alpha_{1}-\ldots-\alpha_{n}\right)$.

Applying observations (1)-(3) and the fact that $H \hat{f}(s)=(-i \pi) \operatorname{sgn} s \hat{f}(s)$ where $H f(x)=p \cdot v \cdot \int \frac{f(y)}{x-y} d y$, we see that the zero commutators are of the form $\left[\prod_{j=1}^{n-k} a_{j}\right]\left[H\left(f \prod_{j=k+1}^{n} a_{j}\right)\right] \in M_{n}$.

The inductive hypothesis is the following: for $\bar{m}=\left(\bar{m}_{1}, \ldots, \bar{m}_{\bar{n}}\right) \in Z^{\bar{n}}$, $0 \leqslant \bar{m}_{g}$ and $|\bar{m}| \leqslant|m|-1$, the symbol $\omega_{\bar{m}} \in M_{\bar{n}}$. From observations (1) $-(3)$ it is clear that we need only show that the inductive hypothesis implies $\omega_{m} \in M_{n}$ when $m=\left(m_{1}, \ldots, m_{n}\right) \in Z^{n}, \sum_{j=1}^{n} m_{j}=|m|$ and $m_{j} \geqslant 1$.

The symbol

$$
\bar{\omega}_{m}=\frac{s R_{-\alpha_{1}}^{m_{1}} \ldots R_{-\alpha_{n}}^{m_{n}} s^{|m|-1} \operatorname{sgn} s}{\prod_{j=1}^{n} \alpha_{j}^{m_{j}}}
$$

is introduced and it is shown that $\omega_{m}-\bar{\omega}_{m}$ is a sum of lower order commutators. It is enough to show that $\bar{\omega}_{m} \in M_{n}$.
(2.1) Defintition. $\mathscr{S}^{x}=\left\{\varphi: \varphi\left(e^{x}\right) \in \mathscr{S}\right\}$.

(2.2) LEMMA. For all $\varphi \in \mathscr{S}^{x}, \sigma \in M_{n}$, and all integers $j, 1 \leqslant j \leqslant n$,
( $\left.\alpha_{j} \mid\right) \sigma(\alpha, s) \in M_{n}$. $\varphi\left(|s| a_{j} \mid\right) \sigma(\alpha, s) \in \mathbb{M}_{n}$.

Proof. For $\varphi \in \mathscr{S}^{x}$,

$$
\varphi(x)=\int_{-\infty}^{\infty} \hat{\psi}(\gamma) x^{i \gamma} d \gamma
$$

where $\psi(x)=\varphi\left(e^{x}\right) \in \mathscr{S}$. Tence

$$
p\left(|s| \alpha_{j} \mid\right) \sigma(\alpha, s)=\int_{-\infty}^{\infty}|s|^{i \gamma}\left|\alpha_{j}\right|^{-i \gamma} \sigma(\alpha, s) \hat{\psi}(\gamma) d \gamma .
$$

But $|s|^{i \gamma}$ is a bounded multiplier with norm not exceeding $(1+|\gamma|)$ so that the operator $T_{r\left(|s| a_{j} \mid\right) \sigma}$ has norm less than or equal to $\int_{-\infty}^{\infty}(1+|\gamma|)^{2}\|\sigma\| \hat{\psi}(\gamma) d \gamma$ where $\|\sigma\|$ is the operator norm of $T_{\sigma}$.

Since $\mathscr{S}$ is closed under the Fourier Transform, $\hat{\psi} \in \mathscr{S}$ and we have $\int_{-\infty}^{\infty}|\hat{\psi}(\gamma)|(1+|\gamma|)^{N} d \gamma<\infty$ for any positive integer $N$.

(2.3) Lmima: Let $\tau: R^{n+1} \rightarrow O$ be a measurable function. Let $\mathscr{C}$ be the set of $\gamma=\left(t_{1}, \ldots, t_{n}\right)$ such that $\left|\alpha_{1}\right|^{t_{1}} \ldots\left|a_{n}\right|^{t_{n}} \tau(\alpha, s) \in M_{n}$. Then $\mathscr{C}$ is a convex set.

Proof. The proof is given in proposition 3 of [6]. Its importance to this paper is the following corollary.

(2.4) Corolrary. Assume that $\omega_{m^{\prime}} \in \mathbb{M}_{n}$ for $\left|m^{\prime}\right|<|m|$. Then for $0 \leqslant \theta_{j} \leqslant 1$ and $\sum_{j=1}^{n} \theta_{j}=1$, we have,

$$
\left|\alpha_{1}\right|^{\theta_{1}} \ldots\left|\alpha_{n}\right|^{0_{n}} \frac{R_{-\alpha_{1}}^{m_{1}} \ldots R_{-a_{n}}^{m_{n}} s^{|m|-1} \operatorname{sgn} s}{\prod_{j=1}^{n} \alpha_{j}^{m_{j}}} \in M_{n} .
$$

Proof. The proof only involves the case $\theta_{1}=1, \theta_{j}=0$ for $j=2, \ldots$ $\ldots, n$. The cases $\theta_{j}=1, \theta_{l}=0$ for $k \neq j$ are tajken care of by a symmetric argument and the rest of the cases follow by convexity from Lemma (2.3). So one must show

$$
\left|a_{j}\right| \frac{R_{-\alpha_{1}}^{m_{1}} \ldots R_{-\alpha_{n}}^{m_{n}} s^{|m|-1} \operatorname{sgn} s}{\prod_{j=1}^{n} \alpha_{j}^{m_{j}}} \in M_{n} .
$$

A simple argumont shows that iff $\sigma(\alpha, s) \in M_{n}$, then $\operatorname{sgn} \alpha_{j} \sigma(\alpha, s) \in M_{n}$. Hence we may replace $\left|\alpha_{j}\right|$ by $\alpha_{j}$ in (2.5). 
If $m_{1}=1$, let $m^{\prime}=\left(m_{2}, m_{3}, \ldots, m_{n}\right)$. Then

$$
\begin{aligned}
& a_{1} \frac{R_{-\alpha_{1}}^{m_{1}} \ldots R_{-\alpha_{n}}^{m_{n}} s^{|m|-1} \operatorname{sgn} s}{\prod_{j=1}^{n} \alpha_{j}^{m_{j}}} \\
& =\frac{R_{-\alpha_{2}}^{m_{2}} \ldots R_{-a_{n}}^{m_{n}}\left(s-\alpha_{1}\right)^{|m|-1} \operatorname{sgn}\left(s-\alpha_{1}\right)}{\prod_{j=2}^{n} \alpha_{j}^{m_{j}}}-\frac{R_{-\alpha_{2}}^{m_{2}} \ldots R_{-\alpha_{n}}^{m_{n}} s^{|m|-1} \operatorname{sgn} s}{\prod_{j=2}^{n} \alpha_{j}^{m_{j}}} .
\end{aligned}
$$

Calling the first term $\sigma_{1}$ and the second term $\sigma_{2}$ we see that $T_{\sigma_{1}}(a, f)$ $=a_{1} T_{\omega_{m^{\prime}}}(a, f)$ and $T_{\sigma_{2}}(a, f)=T_{\omega_{m^{\prime}}}\left(a, a_{1} f\right)$. Both $T_{\sigma_{1}}$ and $T_{\sigma_{2}}$ satisfy Definition (1.3) and so they are both in $M_{n}$.

If $m_{1}>1$, let $m^{\prime}=\left(m_{2}, \ldots, m_{n}\right)$ as above and let $m^{\prime \prime}=\left(m_{1}-1\right.$, $\left.m_{2}, \ldots, m_{n}\right)$. Then once again,

$$
\begin{gathered}
\alpha_{1} \frac{R_{-\alpha_{1}}^{m_{1}} \ldots R_{-a_{n}}^{m_{n}} s^{|m|-1} \operatorname{sgn} s}{\prod_{j=1}^{n} \alpha_{j}^{m} m_{j}}=\frac{R_{-\alpha_{1}}^{m_{1}} R_{-\alpha_{2}}^{m_{2}} \ldots R_{-\alpha_{n}}^{m_{n}} s^{|m|-1} \operatorname{sgn} s}{\alpha_{1}^{m_{1}-1} \prod_{j=2}^{n} \alpha_{j}^{m_{j}}}- \\
-(-1)^{m_{1}-1} \frac{(|m|-1) !}{\left(m_{1}-1\right) !\left|m^{\prime}\right| !} \frac{R_{-\alpha_{2}}^{m_{2}} \ldots R_{-\alpha_{n}}^{m_{n}} s^{\left|m^{\prime}\right|} \operatorname{sgn} s}{\prod_{j=2}^{n} \alpha_{j}^{m_{j}}}
\end{gathered}
$$

The first term is the symbol of the operaton $T_{\omega_{m}{ }^{\prime \prime}}(a, f)$ and the second is a constant times the symbol of $T_{\omega_{m^{\prime}}}\left(a, a_{1} f\right)$. Both of these are commutators of order lower than $|m|$ and so satisfy Definition (1.3) by induction. This proves the corollary.

(2.6) LeMrMa. $\bar{\omega}_{m}-\omega_{m} \in M_{n}$ where

$$
\bar{\omega}_{m}=\frac{s R_{-\alpha_{1}}^{m_{1}} \ldots R_{-\alpha_{n}}^{m_{n}} s^{|m|-1} \operatorname{sgn} s}{\prod_{j=1}^{n} a_{j}^{m_{j}}} .
$$

Proof. Lemma (2.6) follows from the following identity. For $\varphi(s)$ a function with $|m|$ derivatives,

(2.7) $\quad R_{-\alpha_{1}}^{m_{1}} \ldots R_{-\alpha_{n}}^{m_{n}} s \varphi(s)$

Hence,

$$
=s R_{-\alpha_{1}}^{m_{1}} \ldots R_{-a_{n}}^{m_{n}} \varphi(s)-\sum_{j=1}^{n} \alpha_{j} R_{-\alpha_{1}}^{m_{1}} \ldots R_{-a_{j}}^{m_{j}-1} \ldots R_{-\alpha_{n}}^{m_{n_{2}}} \varphi(s) .
$$

$$
\bar{\omega}_{m}-\omega_{m}=\sum_{j=1}^{n} \frac{R_{-\alpha_{1}}^{m_{1}} \ldots R_{-\alpha_{j}}^{m_{j}-1} \ldots R_{-\alpha_{n}}^{m_{n}} s^{|m|-1} \operatorname{sgn} s}{a_{j}^{m_{j}-1} \prod_{\substack{l k \neq j \\ k=1}}^{n} \alpha_{k}^{m_{k}}}
$$

and all of the terms in this sum are in $M_{n}$ since they are the symbols of commutators of order less than $|m|$.
(2.8) Lemara. Let $\eta \in C_{0}^{\infty}$ where $\eta$ is even, non-negative, at most one and satisfies $\eta(t) \equiv 1$ for $t \in[-n, n]$ and $\eta(t) \equiv 0$ for $t \notin[-2 n, 2 n]$. Then $\bar{\omega}_{m}(\alpha, s) \prod_{j=1}^{n}\left(1-\eta\left(s / \alpha_{j}\right)\right) \equiv 0$.

Proof. If $1-\eta\left(s / \alpha_{j}\right) \neq 0$ for all $j$, then $|s|>n\left|\alpha_{j}\right|$ for all $j$ and so $\operatorname{sgn}\left(s-\sum_{j \in J} \alpha_{j}\right)=\operatorname{sgn} s$ for any subset $J$ of $\{1,2, \ldots, n\}$. Thus $\prod_{j=1}^{n}\left(1-\eta\left(s / \alpha_{j}\right)\right)$ $\neq 0$ implies that

$$
\bar{\omega}_{m}(\alpha, s)=s \operatorname{sgn} s \frac{R_{-\alpha_{1}}^{m_{1}} \ldots R_{-\alpha_{n}}^{m_{n}} s^{|m|-1}}{\prod_{j=1}^{n} \alpha_{j}^{m_{j}}}=0 .
$$

Proof of Theorem I. To prove Theorem I it suffices to show that $\bar{\omega}_{m} \in M_{n}$ since by Lemma (2.6) $\bar{\omega}_{m}-\omega_{m} \in M_{n}$. From Lemma (2.8),

$$
\bar{\omega}_{m}(\alpha, s)=-\sum_{J \in \mathscr{J}_{0}}(-1)^{|J|} \prod_{j \in J} \eta\left(s / \alpha_{j}\right) \bar{\omega}_{m}(\alpha, s)
$$

where $\mathscr{J}_{0}$ is the set of non-empty subsets of $\{1,2, \ldots, n\}$ and $|J|$ is the number of elements in $J$. If we define

$$
\omega_{J}(\alpha, s)=\prod_{j \in J} \eta\left(s / \alpha_{j}\right) \bar{\omega}_{m}(\alpha, s),
$$

then

$$
\omega_{J}=\operatorname{sgn} s\left\{\left.\prod_{j \in J} \eta\left(|s| \alpha_{j} \mid\right)|s| \alpha_{j}\right|^{1 /|J|}\right\}\left\{\left[\prod_{j \in J}\left|\alpha_{j}\right|^{1 /|J|}\right] \frac{R_{-\alpha_{1}}^{m_{1}} \ldots R_{-\alpha_{n}}^{m_{n}} s^{|m|-1} \operatorname{sgn} s}{\prod_{\ell=1}^{n} \alpha_{k}^{m_{k}+1}}\right\} .
$$

Let

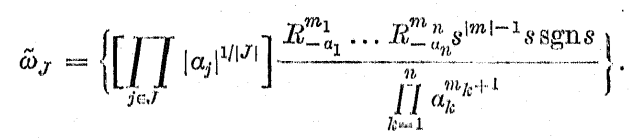

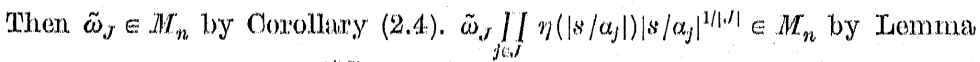
(2.2) and tho fact that $t^{1 / 1, J \mid} \eta(t) \in \mathscr{S}^{n}$. Finally, summing up the $J$ 's wo get that $\bar{\omega}_{m} \in M M_{n}$.

§ 3. The foudamental identity. The purpose of this section is to show that the operators considered in $\$ 2$ can be realized as singular integrals if enough regularity is assumed on the $a_{f}$ 's and $f$. Specitically, for $a_{j} \in \mathscr{D}, j=1,2, \ldots, n, f \in \mathscr{D}, \quad m=\left(m_{1}, \ldots, m_{n}\right)$ and $N=\sum_{j=1}^{n} m_{j}, T_{\omega_{m}}$ 
$=D^{N} H\left[\prod_{j=1}^{n} r_{m_{j}}\left(A_{j} ; x, \cdot\right) f(\cdot)\right]$ and this operator is given by

$$
\frac{1}{e_{m}} p \cdot v \cdot \int \prod_{j=1}^{n}\left\{\frac{r_{m_{j}}\left(A_{j} ; x, y\right)}{(x-y)^{m_{j}}}\right\} \frac{f(y)}{x-y} d y \text { where } \quad e_{m}=\frac{(-i \pi)(-1)^{|m|}}{|m| !(2 \pi)^{n+1}}
$$

(3.1) Lemwa. Assume $g \in \mathscr{D}$ and $x \in R$.

(1) If $N$ is a positive integer and $g(x)=g^{(1)}(x)=\ldots=g^{(N-1)}(x)=0$,

(3.2) $\left(\frac{d}{d x}\right)^{N}\left\{p \cdot v \cdot \int \frac{g(y)}{x-y} d y\right\}=(-1)^{N} N ! p \cdot v \cdot \int \frac{g(y)}{(x-y)^{N+1}} d y$.

(2) If $k, N$ are positive integers with $k \leqslant N$, then (3.3)

$$
\left(\frac{d}{d x}\right)^{N}\left\{p \cdot v \cdot \int \frac{g(y)(x-y)^{k}}{(x-y)} d y\right\}=\frac{(-1)^{k} N !}{(N-k) !}\left(\frac{d}{d x}\right)^{N-l o}\left\{p \cdot v \cdot \int \frac{g(y)}{x-y} \cdot d y\right\} .
$$

Proof. Choose $M>0$ large enough so that the interval [ $-M C, M I]$ contains the support of $g$. Let $\eta \in \mathscr{D}$ be an even function where $0 \leqslant \eta(t) \leqslant 1$, $\eta(t) \equiv 1$ for $t \in[-4 M, 4 M]$ and $\eta(t) \equiv 0$ for $t \notin[-8 M, 8 M]$. Let $\eta_{k}(t)$ $=\eta(t / k)$. For $k \geqslant 1$ we clearly have for $x \in[-2 M, 2 M]$

$$
p . v . \int \frac{g(y)}{x-y} d y=\int \frac{g(y)-g(x)}{x-y} \eta_{l}(x-y) d y .
$$

By differentiating $N$ times and letting $k \rightarrow \infty$ we get the formula

$$
\left(\frac{d}{d x}\right)^{N}\left\{p \cdot v \cdot \int \frac{g(y)}{(x-y)} d y\right\}=(-1)^{N} N ! \lim _{\delta \rightarrow \infty} \int_{|x-y| \leqslant \delta} \frac{g(y)-\sum_{j=1}^{N} \frac{g^{(j)}(x)(y-x)^{j}}{j !}}{(x-y)^{N+1}} d y .
$$

If $g(x)=g^{(1)}(x)=\ldots=g^{(N-1)}(x)=0$, formula $(3.4)$ yields

$$
\lim _{\delta \rightarrow \infty} \int_{|x-y| \leqslant \delta} \frac{g(y)-\frac{g^{(N)}(x)(y-x)^{N}}{n !}}{(x-y)^{N+1}} d y=p \cdot v \cdot \int \frac{g(y)^{\natural}}{(x-y)^{N+1}} d y
$$

which establishes (3.2).

Applying (3.4) to the function $\psi(y)=g(y)(x-y)^{k}$ (where $76, N$ are positive integers with $\left.l_{6} \leqslant . N\right)$ establishes $(3.3)$.

(3.5) Remark. Assume $A_{1}, \ldots, A_{n} \in \mathscr{S}$. Then

$$
\left.\left(\frac{d}{d y}\right)^{N} \prod_{j=1}^{n} r_{m_{j}}\left(A_{j} ; x, y\right)\right|_{y=x}=0
$$

for $N=0,1, \ldots,|m|-1$ where $|m|=\sum_{j=1}^{n} m_{j}$.

Remark (3.5) and Lemma (3.1) show that

$$
\begin{aligned}
D^{|m|} H\left[\prod_{j=1}^{n} r_{m_{j}}\left(A_{j} ; x, \cdot\right) f(\cdot)\right] \\
\quad=(-1)^{|m|}|m| ! \text { p.v. } \int \prod_{j=1}^{n}\left\{\frac{r_{m_{j}}\left(A_{j} ; x, y\right)}{(x-y)^{m_{j}}}\right\} \frac{f(y)}{x-y} d y .
\end{aligned}
$$

(3.6) Lismar. Assume $f \in \mathscr{D}, A_{1}, \ldots, A_{n} \in \mathscr{S}$. Then

(3.7) $\quad i^{|m|} O_{m} \int_{I^{n+1}} X_{(-n)}^{(m)} s^{|m|} \operatorname{sgn} s \hat{A}(\alpha) \hat{f}\left(s-\sum_{j=1}^{n} \alpha_{j}\right) e^{i s x} d \alpha d s$

$$
=p \cdot v \cdot \int \prod_{j=1}^{n}\left\{\frac{r_{m_{j}}\left(A_{j} ; x, y\right)}{(x-y)^{m_{j}+1}}\right\} \frac{f(y)}{x-y} d y
$$

where $O_{m}$ is as in Theorem II.

Proof. To show this we introduce some multiple index notation:

$\mathscr{J}=$ set of subsets of $\{1,2, \ldots, n\}$,

$J^{\prime}=$ complement of $\mathcal{J}$ in $\{1,2, \ldots, n\}$,

$|J|=$ number of elements in $J$,

$k_{J}=\left(k_{j_{1}}, \ldots, k_{j_{t}}\right)$ where $J=\left\{j_{1}, \ldots, j_{t}\right\}$ and $j_{1}<j_{2}<\ldots<j_{t}$,

$K_{J}=\left\{h_{J}: 0 \leqslant h_{i_{j_{1}}} \leqslant m_{j_{1}}-1 ; \ldots ; 0 \leqslant h_{i_{j}} \leqslant m_{j_{t}}-1\right\}$,

$k_{j}^{k} !=k_{j_{1}} ! k_{j_{2}} ! \ldots k_{j_{j}} !$

$\left|h_{J}\right|=k_{j_{1}}+h_{j_{j_{2}}}+\ldots+\ldots+h_{j_{t}}$,

$g^{\left(l k_{j}\right)}(x)=g^{\left(k_{j_{1}}+\cdots++k_{j_{t}}\right)}(x)$.

Using this notation we have the expansion for any $g \in \mathscr{S}$

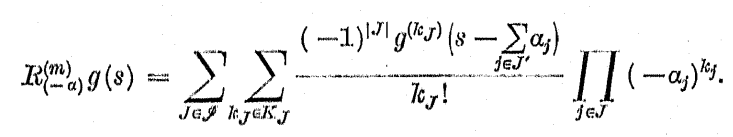

For $\alpha \in R^{n}, \infty \in R$, wo use the following notation:

and

$$
\begin{aligned}
\hat{A}_{J}(\alpha) & =\prod_{j \in J} \hat{A}_{J J}\left(a_{j}\right), \\
A_{J}^{\left(k_{J}\right)}(x) & =\prod_{j \in J} A_{j}^{\left(h_{j}\right)}(x)
\end{aligned}
$$

$$
d \alpha=\prod_{j \in J} d \alpha_{j} .
$$


With this additional notation, setting $g(s)=s^{|m|}$ sgn $s$ and using (3.8)

$$
\begin{gathered}
\int_{R^{n+1}} R_{(-\alpha)}^{(m)} s^{|m|} \operatorname{sgn} s \hat{A}(\alpha) \hat{f}\left(s-\sum_{j \in 1}^{n} \alpha_{j}\right) e^{i s x} d \alpha d s \\
=\sum_{J \in \mathscr{J}^{\prime}} \sum_{k_{J} \in K_{J}} \frac{(-1)^{|m|-\left|k_{J}\right|+|J|}}{(-i \pi)} \cdot \frac{i^{|m|}|m| !}{\left(|m|-\left|k_{J}\right|\right) !} \int_{T^{\prime}} e^{i s x} \times \\
\times \int_{R^{\left|J^{\prime}\right|}}\left[i\left(s-\sum_{j \in J} \alpha_{j}\right)\right]^{|m|-\left|k_{J}\right|}(-i \pi) \operatorname{sgn}\left(s-\sum_{j \in J}^{Y} \alpha_{j}\right) \hat{A}_{J^{\prime}}(\alpha) d \alpha_{J^{\prime}} \times \\
\times \int_{R^{|J|}} \frac{A_{J}^{\left(k_{J} J\right)^{\wedge}}(\alpha)}{k_{J_{J}} !} \hat{f}\left(s-\sum_{j=1}^{n} \alpha_{j}\right) d \alpha_{J} d s .
\end{gathered}
$$

Integrating in $\alpha_{J^{\prime}}$ the inner integral equals

$$
(2 \pi)^{|J|}\left\{\frac{f A_{J}^{(k c J)}}{l_{J} !}\right\}^{\wedge}\left(s-\sum_{j \in J} \alpha_{j}\right) .
$$

Integrating next in $\alpha_{J^{\prime}}$ we get the middle integral is equal to

$$
(2 \pi)^{n}\left\{A_{J}, D^{|m|-\left|k_{J}\right|} H\left(\frac{f A_{J}^{(k J)}}{k_{J} !}\right)\right\}^{\wedge}(s) .
$$

Integrating in $s$ and using Lemma (3.1) and Remark (3.5), the righthand side of (3.9) becomes

$$
\begin{gathered}
\sum_{\sum_{\mathcal{J}}} \sum_{k_{J} \in K_{J}} \frac{(-i)^{|m|}(2 \pi)^{n+1}}{-i \pi}(-1)^{|J|} \prod_{j \in \mathcal{J}} A_{j}(x) D^{|m|} H\left\{f(\cdot) \prod_{j \in J} \frac{A_{j}^{\left(k_{j}\right)}(\cdot)}{7_{k_{j}} !}(x-\cdot)^{k_{k_{j}}}\right\}(x) \\
=i^{|m|} C_{m} p \cdot v \cdot \int \frac{\prod_{j=1}^{n} r_{m_{j}}\left(A_{j} ; x, y\right)}{(x-y)^{|m|+1}} f(y) d y .
\end{gathered}
$$

Proof of Theorem II. Let

$$
I(\varepsilon)=i^{|m|} O_{m} \int_{R^{n+1}} e^{i s x} \frac{R_{(-\alpha)}^{(m)} s^{|m|} \operatorname{sgn} s}{\prod_{j=1}^{n}\left(i \alpha_{j}+\varepsilon\right)^{m_{j}}} \hat{a}(\alpha) \hat{f}\left(s-\sum_{j=m 1}^{n} \alpha_{j}\right) d \alpha d s .
$$

If $A_{j}^{\varepsilon}(x)=e^{-\varepsilon x} \int_{-\infty}^{x} \int_{-\infty}^{t_{1}} \ldots \int_{-\infty}^{t_{m_{j}-1}} a_{j}(s) e^{\varepsilon s} d s$, then $A_{j}^{\varepsilon} \in \mathscr{S}$ and $A_{j}^{s}\left(\alpha_{j}\right)$ $=\frac{\hat{a}_{j}\left(a_{j}\right)}{\left(i a_{j}+\varepsilon\right)^{m_{j}}} \cdot$ Applying Lemma (3.6)

$$
I(\varepsilon)=p \cdot v \cdot \int \prod_{j=1}^{n}\left\{\frac{r_{m_{j}}\left(A_{j}^{\varepsilon} ; x, y\right)}{(x-y)^{m_{j}}}\right\} \frac{f(y)}{x-y} d y .
$$

Since

$$
R_{(-\alpha)}^{(m)} g(s)=\frac{1}{(m-1) !} \int_{0}^{-u_{1}} \cdots \int_{0}^{-\alpha_{n}} g^{(|m|)}\left(s-\sum_{i=1}^{n} u_{j}\right) \prod_{j=1}^{n} u_{j}^{m_{j}-1} d u_{j}
$$

(where $\left.(m-1) !=\left(m_{1}-1\right) ! \ldots\left(m_{n}-1\right) !\right)$, for any $g$ with $|m|$ derivatives, and since $\left(\frac{d}{d s}\right)^{|m|} s^{|m|} \operatorname{sgn} s=|m|$ ! sgns we have the estimate

$$
\left|R_{(-\alpha)}^{(m)} s^{|m|} \operatorname{sgn} s\right| \leqslant \frac{|m| !}{(m-1) !} \prod_{j=1}^{n}\left|\alpha_{j}\right|^{m_{j}} .
$$

Applying the Idebsegue dominated convergence theorem to $I(\varepsilon)$ we seo

$$
\lim _{s \rightarrow 0} I(\varepsilon)=C_{m} \int_{R^{n+1+1}} e^{i s x} \frac{R_{(-\alpha)}^{(m)} s^{|m|} \mathrm{Ngn} s}{\prod_{j=1}^{n} \alpha_{j}^{m_{j}}} \hat{a}(\alpha) \hat{f}\left(s-\sum_{j=1}^{n} \alpha_{j}\right) d a d s .
$$

Finally, as $\varepsilon \rightarrow 0, A_{j}^{g}(x) \rightarrow A_{j}(x), 1 \leqslant j \leqslant n$, uniformly on all compact. sets, and the same is true of the derivatives of $A_{j}^{\varepsilon}(x)$. It follows that

$$
\begin{aligned}
\lim _{s \rightarrow 0} p . v . \int \prod_{j=1}^{n}\left\{\frac{r_{m_{j}}\left(A_{j}^{g} ; x, y\right)}{(x-y)^{m_{j}}}\right\} & \frac{f(y)}{(x-y} d y \\
& =p \cdot v \cdot \int \prod_{j=1}^{n}\left\{\frac{r_{m_{j}}\left(A_{j} ; x, y\right)}{(x-y)^{m_{j}}}\right\} \frac{f(y)}{x-y} d y .
\end{aligned}
$$

\$1. Real variable methods. In this section real variable methods. are introduced to extend the estimates of $\$ 2$ to a full range of $L^{p}$ functions. The truncated opcrator $T_{s}^{m l}(a, f)$ is introduced along with its associated maximal function $T_{*}^{m}$. A good $\lambda$ inequality is proved showing that $T_{*}^{m}$ is bounded in $L^{\alpha}$ by the $L^{q}$ norm of an appropriate product of HardyLittlewood maximal functions.

Somo moro notation and definitions must be introduced. Adopting the notation of $\$ 1$, for $p=\left(p_{1}, \ldots, p_{n}\right)$ and $\left(p, p_{0}\right)=\left(p_{1}, \ldots, p_{n}, p_{0}\right)$. $L^{\left(p, p_{0}\right)}$ and $\|(a, f)\|_{\left(p, p_{0}\right)}$ are defined as before. In addition we define:

$$
\begin{aligned}
& \|a\|_{(p)}=\prod_{j \operatorname{mow} 1}^{n}\left\|a_{j}\right\|_{p, j}, \\
& a_{f}^{*}(x)=\sup _{x \operatorname{san}} \frac{1}{|I|} \int_{I}\left|a_{j}(t)\right| d t
\end{aligned}
$$

where $I$ is an interval and $|X|$ is its length. Wo introduce tho corresponding: multi-index maximal functions,

$$
a^{(*)}(x)=\prod_{j=\infty}^{n} a_{1 j}^{*}(x)
$$


and

$$
(a, f)^{(*)}(x)=\left\{\prod_{j=1}^{n} a_{j}^{*}(x)\right\} f^{*}(x)
$$

Next define the truncated operator

$$
T_{s}^{m}(a, f)(x)=\int_{|x-y|>8} t_{m}(x, y) f(y) d y
$$

where

$t_{m}(x, y)=\frac{1}{x-y} \prod_{j=1}^{n} \frac{r_{m_{j}}\left(A_{j} ; x, y\right)}{(x-y)^{m_{j}}}$ and $T_{*}^{m n}(a, f)(x)=\sup _{a>0}\left|T_{s}^{m}(a, f)(x)\right|$.

We are now ready to state the principal result of this section.

(4.1) Propostmion. If $a_{j} \in L^{1}$ for $j=1, \ldots, n, f \in I^{1}$, then there exists $\gamma_{0}$ so that for $\gamma<\gamma_{0}$,

$\left|x: T_{*}^{m}(a, f)(x)>2 \lambda,(a, f)^{(*)}(x) \leqslant \gamma \lambda\right| \leqslant c \gamma^{n+1}\left|x: T_{*}^{m}(a, f)(x)>\lambda\right|$.

(The notation $\mid x:$ "..." means the measure of the set $x$ such that "...".)

Theorem 1 will follow as an immediate consequence of Proposition (4.1). The proof will be given in a series of lemmas, following closely the real variable methods used by Coifman and Meyer in [5].

(4.2) Leindra. If $|x-y|>\varepsilon,\left|x-x_{1}\right|<\varepsilon / 4$, then for $x_{2} \in(x-\varepsilon / 4$, $x+\varepsilon / 4)$ we have the following estimates.

(b)

$$
\left|t_{m}(x, y)-t_{m}\left(x_{1}, y\right)\right|<c \frac{a^{(*)}\left(x_{2}\right)\left|x-x_{1}\right|}{|x-y|^{2}},
$$

$$
\left|\int_{|x-y|>\varepsilon}\left[t_{m}(x, y)-t_{m}\left(x_{1}, y\right)\right] f(y) d y \leqslant c(a, f)^{(*)}\left(x_{2}\right)\right| .
$$

and for $\left|y-y_{1}\right|<\frac{1}{2}|x-y|,\left|y-y_{2}\right|<\frac{1}{2}|x-y|$,

(c)

$$
\left|t_{m}(x, y)-t_{m}\left(x, y_{1}\right)\right| \leqslant c a^{(*)}\left(y_{2}\right) \frac{\left|y-y_{1}\right|}{|x-y|^{2}} .
$$

Proof.

$$
\begin{aligned}
& t_{m}(x, y)-t_{m}\left(x_{1}, y\right)=\prod_{j=1}^{n} r_{m_{j}}\left(A_{j} ; x, y\right)\left[\frac{1}{(x-y)^{|m|+1}}-\frac{1}{\left(x_{1}-y\right)^{|m|+1}}\right]+ \\
& +\frac{1}{\left(x_{1}-y\right)^{|m|+1}} \sum_{j=1}^{n}\left[r_{m_{j}}\left(A_{j} ; x, y\right)-r_{m_{j}}\left(A_{j} ; x_{1}, y\right)\right] \prod_{l_{k}<j} r_{m_{l k}}\left(A_{l a} ; x, y\right) \times \\
& \times \prod_{l c>j} r_{m_{k}}\left(A_{l k} ; x_{1}, y\right) \\
& \leqslant c a^{(*)}\left(x_{2}\right) \frac{\left|x-x_{1}\right|}{|x-y|^{2}}+c \sum_{j=1}^{n} a_{j}^{*}\left(x_{2}\right) \frac{\left|x-x_{1}\right|}{|x-y|^{2}} \prod_{k \neq j} a_{k}^{*}\left(x_{2}\right)
\end{aligned}
$$

$$
\leqslant e a^{(*)}\left(x_{2}\right) \frac{\left|x-x_{1}\right|}{|x-y|^{2}} .
$$

A standard argument tolls us that for $\left|x-x_{2}\right| \leqslant\left|x-x_{1}\right|$,

$$
\int_{|x-y|>2\left|x-a_{1}\right|} \frac{\left|x-x_{1}\right|}{|x-y|^{2}}|f(y)| d y \leqslant c f^{*}\left(x_{2}\right)
$$

which implios part (b) of Isemma (4.2). Finally, part (c) follows by the same argumont as (a).

Before stating the next lemma it is necessary to introduce a HardyLittlewood maximal function and an appropriate multi-index notation For $(a, f)=\left(a_{1}, a_{2}, \ldots, a_{n}, f\right)$ define

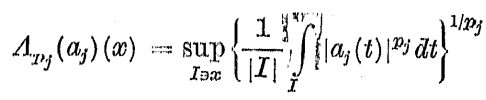

and the corresponding multi-index notation

$$
\begin{gathered}
\Lambda_{(p)}(a)(x)=\prod_{j=1}^{n} \Lambda_{p_{j}}\left(a_{j}\right)(x), \\
\Lambda_{\left(p, p_{0}\right)}(a, f)(x)=\Lambda_{(p)}(a) \Lambda_{p_{0}}(f)(x) .
\end{gathered}
$$

(4.3) Lixarar. Assume $(a, f) \in L^{\left(2, p_{0}\right)}, p_{j} \geqslant 1, \quad j=1,2, \ldots, n$ and $1 \leqslant p_{0}<\infty$. Wurther, assume $0<\delta<q$ where $1 / q=\sum_{j=0}^{n}\left(\mathcal{I} / p_{j}\right)$. If $I^{m}(a, f)$ satisfies the weak type inequality:

$$
\left|x: T^{m}(\alpha, f)(x)>\lambda\right| \leqslant 0\left[\frac{\|(a, f)\|_{\left(p, p_{0}\right)}}{\lambda}\right]^{\alpha},
$$

then the maximat funotion $T_{*}^{m}(a, f)$ satisfies

$$
T_{*}^{m}(a, f)(x) \leqslant o\left[\Lambda_{\delta}\left(T^{m}(a, f)\right)(x)+\Lambda_{\left(p, p_{0}\right)}(a, f)(x)\right]
$$

and the same weak type inequality as in (4.4) is valid for $I_{*}^{m}(a, f)$.

Proof. Beforo proving Lomma (4.3) wo noto that Theorems $\mathrm{I}$ and II imply (4.4) if $\sum_{j=(1)}^{n}\left(\frac{1}{p_{j}}\right)<1,1<p_{j}<\infty$ and the $a_{j}$ 's and $f$ are $C^{\infty}$ with compact support.

The lemma is proved for $a_{j} \in \mathscr{D}, j=1, \ldots, n, f \in \mathscr{D}$ and then it follows from standard. arguments that the lemma can be extended to any $(a, f) \in T^{\left(p, p_{0}\right)}$.

For $\varepsilon>0$, let $\chi_{\theta}$ be the charucteristic function of the interval $[x-\varepsilon$, $x+\varepsilon]$. Let $\eta_{e} \in \mathscr{D}$ be a function which is one on the interval $[x-e, x+e]$, 
less than or equal to one, non-negative and everywhere vanishing outside the interval $[x-2 \varepsilon, x+2 \varepsilon]$. Then, for $x_{1} \in[x-\varepsilon / 4, x+\varepsilon / 4]$, we get the identity

$$
\begin{aligned}
T_{\varepsilon}^{m}(a, f)(x)=\int[ & \left.t_{m}(x, y)-t_{m}\left(x_{1}, y\right)\right]\left[1-\eta_{s}(y)\right] f(y) d y+ \\
& +\int t_{m}\left(x_{1}, y\right) f(y) d y-\int t_{m}\left(x_{1}, y\right) \eta_{s}(y) f(y) d y+ \\
& +\int t_{m}(x, y)\left[\eta_{\varepsilon}(y)-\chi_{\varepsilon}(y)\right] f(y) d y
\end{aligned}
$$

Taking absolute values, raising to the $\delta$ power, averaging in $x_{1}$ over the interval $[x-\varepsilon / 4, x+\varepsilon / 4]$ and taking the $1 / \delta$ power gives the estimate

(4.6) $\left|T^{m}(a, f)(x)\right|$

$$
\leqslant c\left\{(a, f)^{(*)}(x)+\Lambda_{\delta}\left(T^{m}(a, f)\right)(x)+\sup _{\varepsilon>0}\left(\frac{2}{\varepsilon} \int_{x-\varepsilon / 4}^{x+\varepsilon / 4}\left|T^{m}\left(a, \eta_{s} f\right)\left(x_{1}\right)\right|^{\delta} d x_{1}\right)^{1 / \delta}\right\} .
$$

The $(a, f)^{(*)}(x)$ comes from the first and last torm of (4.5) after applying Lemma (4.2). That is,

$$
\begin{aligned}
\mid \int\left[t_{m}(x, y)\right. & \left.-t_{m}\left(x_{1}, y\right)\right]\left[1-\eta_{s}(y)\right] f(y) d y \mid \\
& \leqslant \int\left|t_{m}(x, y)-t_{m}\left(x_{1}, y\right)\right|\left[1-\chi_{s}(y)\right]|f(y)| d y \leqslant c(a, f)^{(*)}(x)
\end{aligned}
$$

and

$$
\begin{aligned}
\mid \int t_{m}\left(x_{1}, y\right)\left[\eta_{\varepsilon}(y)\right. & \left.-\chi_{\varepsilon}(y)\right] f(y) d y \mid \\
& \leqslant c a^{(*)}(x) \int_{2 \varepsilon>|x-y|>\varepsilon} \frac{\left|x-x_{1}\right|}{|x-y|^{2}}|f(y)| d y \leqslant c(a, f)^{(*)}(x) .
\end{aligned}
$$

Both estimates are independent of $x_{1}$ and are left unaffected by averaging in $x_{1}$.

To evaluate the second term on the right hand side of (4.6) we use the fact that the usual Hardy-Littlewood maximal function is weals type 1-1. If we set $E_{\lambda}=\left\{x:\left|T^{m}(a, f)(x)\right|^{\delta^{*}}>\lambda^{\delta}\right\}$, then

$$
\left|E_{\lambda}\right| \leqslant \frac{2}{\lambda^{\delta}} \int_{\mathbb{E}_{\lambda}}\left|T^{m}(a, f)(x)\right|^{\delta} d x
$$

using Kolmogorov's inequality

$$
\leqslant \frac{2}{\lambda^{\delta}}|W|^{1-\delta / \alpha}\|(a, f)\|_{\left(p, p_{0}\right)}^{\delta} .
$$

Taking the $1 / \delta$ power of both sides and simplifying we get

$$
\left|E_{\lambda}\right| \leqslant\left\{\frac{2\|(\alpha, f)\|_{\left(p, p_{0}\right)}}{\lambda}\right\}^{q}
$$

To evaluate the last term in (4.6) we need to be a little careful. We let $\eta_{2 \varepsilon}=\eta_{s}(t / 2)$. Then $\eta_{2 \varepsilon}(t) \equiv 1$ for $|t-x|<2 \varepsilon$. If we adopt the notation $\eta_{28} a=\left(\eta_{28} a_{1}, \ldots, \eta_{2 s} a_{n}\right)$, then for $\left|x_{1}-x\right|<\varepsilon / 4$,

$$
T^{m}\left(a, \eta_{B} f\right)\left(\infty_{1}\right)=T^{m}\left(\eta_{2 \varepsilon} a, \eta_{B} f\right)\left(x_{1}\right)
$$

Applying Kolmogorov's inequality again (the weak type property is guaranteed since $\eta_{2 s} a_{j} \in \mathscr{D}$ and $\left.\eta_{s} f \in \mathscr{D}\right)$ wo have,

$$
\begin{aligned}
& \left\{\frac{2}{\varepsilon} \int_{\alpha-8 / 4}^{x++/ / 4}\left|T^{m}\left(\eta_{2 s} a, \eta_{s} f\right)\left(x_{1}\right)\right|^{\delta} d x_{1}\right\}^{1 / \delta} \leqslant\left\{\frac{2}{\varepsilon} c_{\delta}\left(\frac{\varepsilon}{2}\right)^{\delta / \alpha}\left\|\left(\eta_{2 \beta} a, \eta_{\varepsilon} f\right)\right\|_{\left(p, p_{0}\right)}^{\delta}\right\}^{1 / \delta} \\
& \leqslant c \prod_{j=1}^{n}\left\{\frac{1}{8 \varepsilon} \int_{x=4 \varepsilon}^{x+4 s}\left|a_{j}(t)\right|^{p_{j} j} d t\right\}^{1 / p_{j}}\left\{\frac{1}{4 \varepsilon} \int_{x-2 \varepsilon}^{x+2 \varepsilon}|f(t)|^{p_{0}} d t\right\}^{1 / p_{0}} \leqslant c \Lambda_{\left(p, p_{0}\right)}(a, f)(x) \text {. }
\end{aligned}
$$

Since $\delta<\delta_{1} \Rightarrow \Lambda_{\delta}(f)(x) \leqslant \Lambda_{\delta_{1}}(f)(x)$ we get the estimate

$$
\left|T_{*}^{m}(a, f)(x)\right| \leqslant c\left[\Lambda_{\delta}\left(T^{m}(a, f)(x)\right)+\Lambda_{\left(p, p_{0}\right)}(a, f)(x)\right] .
$$

The weak type estimate for $T_{*}^{m}$ then follows from (4.7) and

(4.8) Onsturvatron. If $T_{1}, \ldots, T_{n}$ are weat type $\left(p_{j}, p_{j}\right)$ operators with $1 / q=\sum_{j=1}^{n}\left(1 / p_{j}\right)$ and $\infty>p_{j} \geqslant 1$ for $j=1,2, \ldots, n$, then $\prod_{j=1}^{n}\left|T_{j} a_{j}\right|$ satisfies the weak estimate of (4.4:).

(4.9) Limmana. If $(a, f) \in L^{\left(p, p_{0}\right)}$, where $1 / q=\sum_{j=0}^{n}\left(1 / p_{j}\right)$, and $(4.4)$ is satisfied for this set of $p_{j}$ 's, then there exist constants $\gamma_{0}>0$ and $c>0$ so that for $0<\gamma<\gamma_{0}$

$(4.1 .0) \quad\left|x: T_{*}^{m}(a, f)(x)>2 \lambda, \Lambda_{\left(p, p_{0}\right)}(a, f)(x) \leqslant \gamma \lambda\right|$

$$
\leqslant c \gamma^{\alpha}\left|x: T_{*}^{m}(a, f)(x)>\lambda\right| \text {. }
$$

Proof. $\left\{x: T_{*}^{m}(a, f)(x)>\lambda\right\}=\bigcup_{j} X_{j}$ where the $I_{j}$ 's are open disjoint intervals, $I_{j}:=\left(\alpha_{j}, \alpha_{j}+\delta_{j}\right)$ and $I_{*}^{m b}(a, f)\left(\alpha_{j}\right) \leqslant \lambda$ since $\alpha_{j} \notin \bigcup_{j} I_{j}$.

It will suffice to establish (4.10) for each $I_{j}$ since the $I_{j}$ 's are disjoint. Ohoose an $I_{j}$ with a point $z$ wattisfying $\Lambda_{\left(p, y_{0}\right)}(a, f)(z) \leqslant \gamma \lambda$. If $I_{j}$ contains no snch point, $(4.10)$ is automatically satisfied. Let $\bar{I}_{j}=\left(\alpha_{j}-2 \delta_{j}, \alpha_{j}+2 \delta_{j}\right)$,

$$
f_{1}=\chi_{\tilde{I}_{j}} f \quad\left(\chi_{\tilde{I}_{j}} \text { is the chameteristic function of the interval } \tilde{I}_{j}\right) \text {, }
$$

$$
f_{2}=f-f_{1} .
$$

For $x \in I_{j,}, T_{*}^{m}\left(a, f_{1}\right)(x)=T_{*}^{m}\left(\chi_{\bar{I}_{j}} a, \chi_{\bar{x}_{j}} f\right)(x)$. By virtuo of Lemma (4.3) 
we have for $1 / q=\sum_{j=0}^{n}\left(1 / p_{j}\right)$,

(4.11) $\left|x \in I_{j}: T_{*}^{m}\left(\chi_{\bar{I}_{j}} a, \chi_{\bar{I}_{j}} f\right)(x)>\beta \lambda\right| \leqslant c\left[\frac{\left\|\left(\chi_{\bar{I}_{j}} a, \chi_{\bar{I}_{j}} f\right)\right\|_{\left(p, p_{0}\right)}}{\beta \lambda}\right]^{\alpha}$

$$
\begin{aligned}
& \leqslant c\left[\prod_{k=1}^{n}\left|\bar{I}_{j}\right|^{1 / p_{k}}\left\{\frac{1}{\left|\bar{I}_{j}\right|} \int_{\bar{I}_{j}}\left|a_{l_{k}}(t)\right|^{p_{k}} d t\right\}^{1 / p_{k}}\right]\left[\left|\bar{I}_{j}\right|^{1 / p_{0}}\left\{\frac{1}{\left|\bar{I}_{j}\right|} \int_{\bar{I}_{j}}|f(t)|^{p_{0}} d t\right\}^{1 / p_{0}}\right] \\
& \leqslant c\left\{\frac{A_{\left(p, p_{0}\right)}(a, f)(z)\left|\bar{I}_{j}\right|^{j} \sum_{0}^{n}\left(1 / p_{j}\right)}{\beta \lambda}\right\}^{\alpha} \leqslant c(\gamma / \beta)^{\alpha}\left|I_{j}\right| .
\end{aligned}
$$

For the $f_{2}$ part

(4.12) $\left|T_{s}^{m}\left(a, f_{2}\right)(x)\right| \leqslant\left|T_{s}^{m}\left(a, f_{2}\right)(x)-T_{s}^{m}\left(a, f_{2}\right)\left(\alpha_{j}\right)\right|+\left|T_{s}^{m}\left(a, f_{2}\right)\left(\alpha_{j}\right)\right|$

$$
\begin{aligned}
& \leqslant \lambda+\left|\int\left[t_{m}(x-y)-t_{m}\left(\alpha_{j}, y\right)\right] f_{2}(y) d y\right|+ \\
&+\int_{x-s}^{a_{j}-8}\left|t_{m}(x, y)\right|\left|f_{2}(y)\right| d y+\int_{x+s}^{a_{j}+\varepsilon}\left|t_{m}(x, y)\right|\left|f_{2}(y)\right| d y \\
& \leqslant \lambda+c_{1}(a, f)^{(*)}(z) \leqslant \lambda+c_{1} \gamma \lambda .
\end{aligned}
$$

The estimate for the first integral in (4.12) follows from Lemma (4.2). The estimate for the second and third integral follow from the observation that

$$
\left|t_{m}(x, y)\right| \leqslant c \frac{a^{(*)}(z)}{\left|I_{j}\right|+\varepsilon} .
$$

Combining the $f_{1}$ and $f_{2}$ estimates,

$$
\begin{aligned}
\mid x \in I_{j}: T^{m}\left(a, f_{1}\right)(x)>2 \lambda, A_{\left(p, p_{0}\right)}( & a, f)(x) \leqslant \gamma \lambda \mid \\
& \leqslant\left|x \in I_{j}: T_{*}^{m}\left(a, f_{1}\right)(x)>\lambda\left(1-e_{1} \gamma\right)\right|+ \\
& +\left|x \in I_{j}: T_{*}^{m}\left(a, f_{2}\right)(x)>\lambda\left(1+o_{1} \gamma\right)\right| \\
& \leqslant o\left(\gamma \mid\left(1-c_{1} \gamma\right)\right)^{\alpha}\left|I_{j}\right| .
\end{aligned}
$$

Since the constants $o_{1}, o$ are independent of the choice of $I_{1}$ we can add the estimate for each interval to establish (4.10). $0<q$.

(4.13) CoRoLLARY. $\int\left|T_{*}^{m}(a, f)(x)\right|^{\alpha} d x \leqslant c \int\left|\Lambda_{\left(x, p_{0}\right)}(a, f)(x)\right|^{a} d x$ for any

(4.14) OoROLLARY. For $a \in L^{(\infty)}\left(i . e \cdot a_{j} \in L^{\infty}, j=1, \ldots, n\right)$ and $f \in L^{p_{0}}, p_{0}>1$,

where $\|a\|_{(\infty)}=\prod_{j=1}^{n}\left\|a_{j}\right\|_{\infty}$.

$$
\left\|T_{*}^{m}(a, f)\right\|_{p_{0}} \leqslant c\|a\|_{(\infty)}\|f\|_{p_{0}}
$$

Corollary (4.13) is the standard result of a good $\lambda$ inequality (see remarks at the end of this section). Corollary (4.14) follows from the fact that any function in $L^{\infty}$ is locally in $L^{p}$ and the fact that $A_{p}(f)(x) \leqslant\|f\|_{\infty}$.

To finish the proof of Proposition. (4.1) we need one more lemma which extends the range of $p$ 's.

(4.15) Lisirus. If $a_{j} \in I^{1}$ for $j=1,2, \ldots, n$ and $f \in L^{1}$, then

$$
\left|x: I^{m}(a, f)(x)>\lambda\right| \leqslant c\left\{\frac{\|(a, f)\|_{(1,1)}}{\lambda}\right\}^{1 /(n+1)}
$$

where $\|(a, f)\|_{(1,1)}=\left\{\int_{j=1}^{n}\left\|a_{j}\right\|_{1}\right\}\|f\|_{1}$.

Proof. The proof eonsists of showing the following. For any $j=0,1, \ldots$ $\ldots, n,\left\|a_{1}\right\|_{1}=\left\|a_{2}\right\|_{1}=\ldots=\left\|a_{j}\right\|_{1}=\|: f\|_{1}=1$ and $\left\|a_{j+1}\right\|_{\infty}=\ldots=\left\|a_{n}\right\|_{\infty}=1$, then

$$
\left|x: T^{m}(a, f)(\infty)>\lambda\right| \leqslant \frac{c}{\lambda^{1 /(j+1)}} .
$$

Choosing $j=n$ and using the multilinearity of $T^{m}(a, f),(4.16)$ will imply the lemma. We proceed by induction.

First, the estimate (4.16) is valid for $j=0$ (i.e. for all the $a_{j}$ 's in $L^{\infty}$ ) since by Lemma (4.2)

$$
\left|t_{m}(x, y)-t_{m}\left(x, y_{1}\right)\right| \leqslant c a^{(*)}\left(y_{2}\right) \frac{\left|y-y_{1}\right|}{|x-y|^{2}} \quad \text { for } \quad|x-y|>2\left|y-y_{2}\right|,
$$

and $I^{m}$ is bounded in $t^{2}$ as an operator acting on $f$ whenever all the $a_{j}{ }^{\prime} \mathrm{s}$ are in $L^{\infty}$. By a standard Calderon-Zygmund argument as given in [9] we get that $T^{m}$ is weak type 1-1 as an operator on $f$.

Next assume (4.16) is valid for $1,2, \ldots, j-1$ and assume $\left\|a_{1}\right\|_{1}=\ldots$ $\ldots=\left\|a_{j}\right\|_{1}=\|f\|_{1}=1$ and $\left\|a_{j+1}\right\|_{\infty}=\ldots=\left\|a_{n}\right\|_{\infty}=1$. Let $\Omega=\left\{x: a_{j}^{*}(x)\right.$ $\left.>\lambda^{1 /(j+1)}\right\}$. It follows that $\Omega=\bigcup_{k} I_{l o}$ and $a_{j}=g+b$ where

(i) The $I_{I_{c}}$ 's are open and disjoint.

(ii) $\sum_{l o}\left|I_{l o}\right|<o / \lambda^{1 /(j+1)}$.

(iii) $\frac{1}{\left|I_{j_{k}}\right|} \int_{L_{k}}\left|a_{j}(t)\right| d t \leqslant \lambda^{\mathrm{i} /(j+1)}$

(iv) $|g(x)| \leqslant \lambda^{1 /(j+1)}$.

(v) $b=\sum_{l k} b_{l k}$ where $b_{k}$ is supposted in $x_{k}$.

This follows by defining $g$ by

$$
g(x)= \begin{cases}a_{j}(x), & x \notin \Omega, \\ \frac{1}{\left|I_{k_{i}}\right|} \int_{I_{k}} a_{j}(t) d t, & x \in I_{t c}\end{cases}
$$


and defining $b=a_{j}-g$. Then if $\chi_{I_{h}}$ denotes the characteristic function of the interval $I_{k}$, we define $b_{k}=b \chi_{I_{k}}$.

Let $y_{l_{i}}$ be the center of the inetrval $I_{l_{i}}$ and let $2 I_{l c}$ denote the interval which is twice the length of $I_{l o}$ and also centered at $y_{k 0}$.

For $x \notin 2 I_{l k}$ we observe:

$$
\left|\int_{y}^{x} b_{l o}(t)(x-t)^{m} d t\right| \leqslant 2|x-y|^{m}\left|I_{l_{0}}\right| \lambda^{\lambda^{1 /(j+1)}}
$$

since

$$
\frac{1}{\left|I_{I_{k}}\right|} \int_{I_{l_{k}}}\left|b_{l_{k}}(t)\right| d t \leqslant 2\left\{\frac{1}{\left|I_{k}\right|} \int_{\lambda_{l_{k}}}\left|a_{j}(t)\right| d t\right\} \leqslant 2 \lambda^{1 /(j+1)} .
$$

$$
\left|T^{m}\left(a_{1}, \ldots, a_{j-1}, b_{k}, a_{j+1}, \ldots, a_{n}, f\right)(x)\right|
$$

(3) The map

$$
\leqslant c \prod_{i=1}^{j-1} a_{i}^{*}(x) \prod_{i=j+1}^{n}\left\|a_{i}\right\|_{\infty} \frac{\left|I_{k}\right| \lambda^{\mathrm{I} /(j+1)}}{\left|I_{k_{k}}\right|^{2}+\left|x-y_{k_{c}}\right|^{2}} \int_{Y_{k}}|f(t)| d t .
$$

$$
L: f \rightarrow \sum_{k} \frac{\left|I_{k}\right|}{\left|I_{k}\right|^{2}+\left|x-y_{k}\right|^{2}} \int_{I_{l k}}|f(t)| d t
$$

satisfies the following weak type estimate:

When $f \in L^{1}$,

$$
\left|x \notin \bigcup_{k} 2 I_{k}: L f(x)>\lambda\right| \leqslant 0\|f\|_{1} / \lambda .
$$

This follows from using Fubini's theorem to get

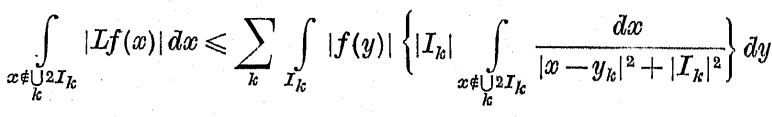

$$
\begin{aligned}
& \leqslant c \int_{\Omega}|f(y)| d y \leqslant c\|f\|_{1} .
\end{aligned}
$$

This strong type result implies the weak estimate (4.17). Putting (1), (2) and (3) together we see that for $x \notin \bigcup_{l c} 2 I_{k}$,

$\left|T\left(a_{1}, \ldots, a_{j-1}, b, a_{j+1}, \ldots, a_{n}, f\right)(x)\right| \leqslant c a_{1}^{*}(x), \ldots, a_{j-1}^{*}(x) \lambda^{1 /(j+1)} L f(x)$.

So we have the product of $j$ operators which are all weak type $(1,1)$ and the functions $a_{1}, \ldots, a_{j-1}, f$ all have an $L^{1}$ norm of one. This implies that

$$
\begin{aligned}
\mid x \notin \bigcup_{k} 2 I_{k}: & T^{m}\left(a_{1}, \ldots, a_{j-1}, b, a_{j+1}, \ldots, a_{n}, f\right)(x)>\lambda \mid \\
& \leqslant\left|x \notin \bigcup 2 I_{k}: c a_{1}^{*}(x), \ldots, a_{j-1}^{*}(x) L_{f} f(x)>\lambda^{j /(j+1)}\right| \\
& \leqslant c / \lambda^{1 /(j+1)} .
\end{aligned}
$$

This follows from observation (4.8) about weak type estimates for the product of weak type operators.
Wo next use the inductive hypothesis to make a weals type estimate for $y$.

$$
\begin{aligned}
& \left|x:, I^{m n}\left(a_{1}, \ldots, a_{j \cdots 1}, y, a_{j+1}, \ldots, a_{n}, f\right)(x)>\lambda\right| \\
& =\left|x: I^{m !}\left(a_{1}, \ldots, a_{j-1}, \frac{g}{\|g\|_{\infty}}, a_{j+1}, a_{n}, f\right)(x)>\frac{\lambda}{\|g\|_{\infty}}\right|
\end{aligned}
$$

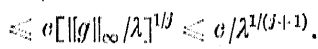

Sineo $\|g\|_{\infty} \therefore \lambda^{1 /(j ! 1)}$, wo haves

(5) There is

$$
\left|\bigcup_{k} 2 I_{b}\right| \leqslant 2|\Omega| \leqslant a \mid \lambda^{1 /(j+1)}
$$

Putiting together estimaties (4.18), (4.19) and (4.20) we have shown

$$
\left|x: T^{m}(a, f)(x)>\lambda\right| \leqslant c / \lambda^{1 /(j+1)} .
$$

If the $L^{1}$ and $T_{I^{\infty}}$ notms of the finnetions are arbitrary, by dividing by the appropriabe norms and nsing the multilinearity of $T^{\text {min }}$ we get the estinatio:

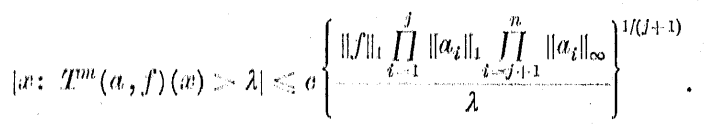

Tu particuliur, for $(a, f)$ \& $T_{t^{(1,1)}}$

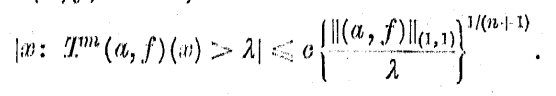

Proof of Theorem IIr. Proposition (4.1) then follows by applying Lemmas (4,3) and (4.9) to Lemma (4.15). In other words we have the estimate:

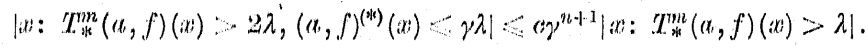

Theorem III then tollows by the following argument. If $a_{j} \in L^{1}$ for $j=1, \ldots, n$ and $f \in L^{1}$, then $(a, f)^{(*)}(x) \simeq \delta /|x|^{n+1}$ as $x \rightarrow \rightarrow \infty$. Theneo $\left.\int_{i} l(a, f)^{(*)}(x)\right]^{q} d x=1<\infty$ for $q<1 /(n+1)$. For $q>1 /(n+1)$, Jemma $(4.3)$

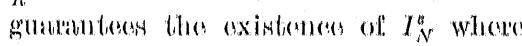

$$
\begin{aligned}
& I_{N}^{n}=q \int_{a}^{N} \lambda^{\eta \cdots-1}\left|m: I_{*}^{m p}(a, f)(x)>\lambda\right| d \lambda
\end{aligned}
$$

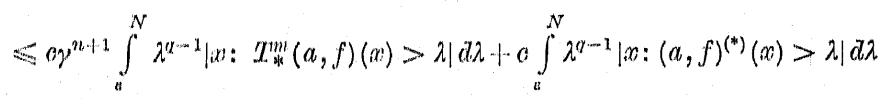

$$
\begin{aligned}
& \leqslant o \gamma^{n+1} I_{N}^{n}+o_{\gamma} \int_{I R}\left[(a, f)^{(*)}(x)\right]^{\mu} d x .
\end{aligned}
$$


Choosing $\gamma$ so that $c \gamma^{n+1} \leqslant \frac{1}{2}$, letting $N \rightarrow \infty$ and $\varepsilon \rightarrow 0$ completes the proof of Theorem III.

By standard argumentis Theorem III implies the following important corollary.

(4.21) Conollanx. For $(a, f) \in L^{\left(n, p_{0}\right)}, \sum_{j=0}^{n}\left(1 / p_{j}\right)=1 / q<n+1,1<$ $p_{0}<\infty, 1<p_{j} \leqslant \infty$, and $T_{i}^{m}(a, f)$ and $T_{*}^{m}(a, f)$ the
in Theorem III, the following propertics are satisfied:
(1) $\left\|T_{*}^{p n}(a, f)\right\|_{q} \leqslant c\|(a, f)\|_{\left(p, p_{0}\right)}$
(2) $\lim _{\varepsilon \rightarrow 0} T_{\varepsilon}^{m}(a, f)(x)$ exists almost everywhore.

\section{References}

[1] B. M. Baishansky and R. Coifman, On singular integrals, Proc. Syrnpos. Pure Math., vol. 10, Amer. Math. Soc., Providence, R. I. (1967), pp. 1-17. [2] A. P. Calderón, Oommutators of singular integrals, Proc. Nit. Acad. Sci. U.S.A. 53 (1965), pp. 1092-1099.

[3] - On algebras of singular integral operators, Proc. Sympos. Puro Math., vol. 10, Amer. Math. Soc., Providence, R. T. (1967), pp. 18-55.

[4] R. Coifm an and C. Fefferman, Weighted norm inequalities for maximal functions and singular integrals, Studia Math. 51 (1974), pp. 241.-250.

[5] R. Coifman and Y. Meyer, On commutators of singular integrals and bilinean singular integrals, Trans. Amer. Math. Soc. 212 (1975), pp. 315 -331.

[6] _- - Oommutateurs d'integrales singulières, Seminaire d'Analyso Harmoniquo, Orsay, France 1976.

[7] -, - Oommutateurs d'integrales singulières et opérateurs multilineaires, Ann. Inst. Fourier, Grenoble 1977.

[8] R. Coifman, R. Rochberg, and G. Weiss, Factorization theorems for ILartly spaces in several variables, Ann. of Math. 103 (1976), pp. 611-635.

[9] E. M. Stein, Singular integrals and differentiability propertios of funotions, Princeton 1970.

\section{An analog of tho Mareinkiewicz integral in ergodic theory}

by

ROC RE R. JONGS (Chicago, ril.)

Alstract. Tot 'T bo su invertible moasure preserving point transformation from a space $X$ onto iliselle. Defines $\tau_{u}(x)=$ int $\left\{n ; 0, T^{n} x \in B\right\}$. The andog of the classical Marcinkiowioz integral $x(f)(x)$, is dofinod by

$$
I(f)(x)=\sum_{k=1}^{\infty} \frac{\tau_{B}\left(I^{\prime / x} x\right) f\left(T^{\prime / x} x\right)}{l^{2}}
$$

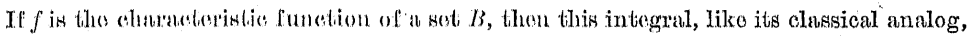

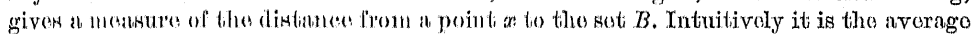
amount of time thes point spendes ontsides the set $B$ during its orbit. It is nsod to give a direct proof that lihe argodic Ifilberti transtorn is woak type $(1,1)$.

Theorems. Inot $(X, \Sigma, m)$ denoto a complete nonatomic probability space, and $T$ an ereolic motsure preserving invertible point transformation from $X$ onto itself. For $B \in \Sigma$, with $0<m(B)<1$ and a point $x$, consider the orbiti, $x, T x, T{ }^{2} x, \ldots$ Wollowing this orbit wo will enter and leave the set $B$ infinitely often. In the following wo will be interested in various messures of: the distance from the point $x$ to the set $B$.

$\Lambda$ natiand mosture is the recurrenes time, defined by

$$
\nu_{l}(x)= \begin{cases}\operatorname{int}\left\{n_{i}>0 \mid T^{n} x \in B\right\}, & x \in B \\ 0, & x \notin B\end{cases}
$$

This function han boen previously studied by Kane [6] and Blum and Rosemblatiti [1]. Kaic has shown that $\left\|\nu_{B}\right\|_{1}=1 / m(B)$, and Blum and Rosanhlatiti have stiodied tha higher momontis.

A second monsure, rotutien to the recurrence time, is defined by

$$
\tau(x): \cdots \inf \left\{n \geq 0 \mid T^{-n} \infty \in B\right\} .
$$

Tht is not hard to seco thati $\tau(x)$ may fail to bo in $L^{1}(X)$. In fact $\tau(x) \in L^{1}(X)$ if and only if: $\nu_{B}(x)$ has a linite second moment.

Both of the above mensumenents are local in the sense that after a return. to $B$, they fail to observe the remainder of the orbit. Howover, 